\title{
Perioperative cerebral ischemia promote infiltrative recurrence in glioblastoma
}

\author{
Anna-Luisa Thiepold ${ }^{1}$, Sebastian Luger ${ }^{2}$, Marlies Wagner ${ }^{3}$, Natalie Filmann ${ }^{4}$, \\ Michael W. Ronellenfitsch ${ }^{1}$, Patrick N. Harter ${ }^{5}$, Anne K. Braczynski ${ }^{5}$, Stephan \\ Dützmann $^{6}$, Elke Hattingen ${ }^{3}$, Joachim P. Steinbach ${ }^{1}$, Christian Senft ${ }^{6}$, Johannes \\ Rieger $^{1}$ and Oliver Bähr ${ }^{1}$ \\ ${ }^{1}$ Dr. Senckenberg Institute of Neurooncology, Goethe-University Hospital, Frankfurt, Germany \\ 2 Department of Neurology, Goethe-University Hospital, Frankfurt, Germany \\ ${ }^{3}$ Institute of Neuroradiology, Goethe-University Hospital, Frankfurt, Germany \\ ${ }^{4}$ Institute of Biostatistics and Mathematical Modeling, Goethe-University Hospital, Frankfurt, Germany \\ ${ }^{5}$ Edinger Institute, Institute of Neurology, Goethe-University Hospital, Frankfurt, Germany \\ ${ }^{6}$ Department of Neurosurgery, Goethe-University Hospital, Frankfurt, Germany \\ Correspondence to: Oliver Bähr, email: oliver.baehr@med.uni-frankfurt.de
}

Keywords: glioblastoma, hypoxia, patterns of progression, perioperative ischemia, MRI

Received: March 24, $2015 \quad$ Accepted: April 11, $2015 \quad$ Published: May 04, 2015

This is an open-access article distributed under the terms of the Creative Commons Attribution License, which permits unrestricted use, distribution, and reproduction in any medium, provided the original author and source are credited.

\section{ABSTRACT}

Background: Hypoxia is a key driver for infiltrative growth in experimental gliomas. It has remained elusive whether tumor hypoxia in glioblastoma patients contributes to distant or diffuse recurrences. We therefore investigated the influence of perioperative cerebral ischemia on patterns of progression in glioblastoma patients.

Methods: We retrospectively screened MRI scans of 245 patients with newly diagnosed glioblastoma undergoing resection for perioperative ischemia near the resection cavity. 46 showed relevant ischemia nearby the resection cavity. A control cohort without perioperative ischemia was generated by a 1:1 matching using an algorithm based on gender, age and adjuvant treatment. Both cohorts were analyzed for patterns of progression by a blinded neuroradiologist.

Results: The percentage of diffuse or distant recurrences at first relapse was significantly higher in the cohort with perioperative ischemia $(61.1 \%)$ compared to the control cohort (19.4\%). The results of the control cohort matched well with historical data. The change in patterns of progression was not associated with a difference in survival.

Conclusions: This study reveals an unrecognized association of perioperative cerebral ischemia with distant or diffuse recurrence in glioblastoma. It is the first clinical study supporting the concept that hypoxia is a key driver of infiltrative tumor growth in glioblastoma patients.

\section{INTRODUCTION}

The course of disease of patients with glioblastoma is almost universally progressive and ultimately fatal. At first recurrence, local re-growth is the most frequent pattern of progression with approximately $80 \%$, whereas diffuse or distant progression is rare. Even at later recurrences local tumor growth remains the predominant pattern with about $71 \%$.[1] Nevertheless, the diffusely infiltrative pattern of glioblastoma growth is one of the most difficult obstacles for next-generation glioblastoma therapies aiming at long-term disease control.

Hypoxia has a fundamental role in the microenvironment of glioblastoma and is known to promote resistance to radio- and chemotherapy. Moreover, hypoxia is a key driver for invasive growth and distant 
metastasis.[2-5] Mechanisms include the detection of hypoxia by hypoxia-inducible-factor (HIF)-1-alpha signaling and the generation of adaptive responses including enhanced migration and invasion that may be mediated by c-MET and other factors.[6-8] That hypoxia is a key driver for invasive glioma growth has been demonstrated in preclinical studies in vitro and in vivo. However, evidence that this is true in glioma patients is missing. In the current study we took advantage of the fact that some glioma patients suffer a cerebral ischemia during resection and thereby presumably exposing residual tumor cells to hypoxia. While antiangiogenic therapies have been extensively studied in glioblastoma, the time course and degree of hypoxia induced by these remain controversial.[9-14] In contrast, extent and time course of cerebral hypoxia caused by ischemic stroke can be much better defined. Since cerebral ischemia can be a complication following tumor resection, mainly by arterial or venous vessel injury during surgery,[15] we performed a retrospective study addressing the association of postoperative ischemia with patterns of recurrence in glioblastoma patients. We hypothesized that perioperative ischemia near the resection cavity might induce a more invasive pattern of recurrence by induction of sublethal hypoxia in residual tumor cells. We therefore screened our local brain tumor database for patients with relevant lesions of restricted diffusion on immediate postoperative MRI. The patterns of recurrence in these patients were analyzed by a blinded neuroradiologist. The results were compared with a cohort of matched pairs of patients without perioperative stroke and historical data.

\section{RESULTS}

\section{Patient characteristics}

Patient characteristics were representative for a cohort of glioblastoma patients undergoing resection (Table 1). All patients with perioperative ischemia had a volume of restricted diffusion of at least $3 \mathrm{~cm}^{3}$. Median volume of restricted diffusion in this cohort was $17.1 \mathrm{~cm}^{3}$ (average $22.3 \mathrm{~cm}^{3}$, range $3.4-61.5 \mathrm{~cm}^{3}$ ).

Distribution of the baseline characteristics including age, Karnofsky performance score (KPS), type of resection, histology and MGMT methylation status were comparable between both groups. Since we also matched for adjuvant treatment until first recurrence these parameters were identical. In both groups, $71.7 \%$ of the patients were treated with radiotherapy plus concomitant and adjuvant temozolomide according to the EORTC 26981/22981-NCIC CE3 study.[16] In some older patients radiotherapy or temozolomide were administered alone. [17]

A longer period between resection and start of adjuvant treatment could be a reasonable factor influencing patterns of progression. Regarding this aspect, we did not find a difference between the both groups (Table 1).

In each group 10 patients were not fully evaluable and regarded as drop-outs. In the control cohort one patient has not progressed yet, 3 patients had insufficient radiology and 6 patients were lost for follow-up. In the ischemia cohort 2 patients had not progressed yet, 2 patients had deceased before progression and 6 patients were lost for follow-up.

\section{Patterns of recurrence}

In each group 36 patients had radiologically confirmed first recurrence and were fully available for analysis of pattern of progression. Recurrences did not involve the area of former ischemia.

22 of 36 patients $(61.1 \%)$ with perioperative ischemia showed an infiltrative pattern of progression (Figure 4A). In detail, diffuse recurrence was observed in 10 of 36 patients $(27.8 \%)$ and distant recurrence in 12 of 36 patients $(33.3 \%)$. Accordingly, local recurrence was only observed in $38.9 \%$ of the patients with perioperative ischemia (14 of 36 patients). In contrast, in the control cohort of matched pairs (patients without perioperative ischemia) only $19.4 \%$ (7 of 36 patients) showed an infiltrative pattern of progression (Figure 4A). Diffuse recurrence was observed in 2 of 36 patients $(5.6 \%)$ and distant recurrence in 5 of 36 patients $(13.9 \%)$. Local recurrence was observed in $80.6 \%$ of the control patients (29 of 36 patients). Notably, the results of the matched pair cohort correspond to previously published data on patterns of progression in patients with glioblastoma.[1, 18, 19]

\section{Competing risk analysis}

To confirm that the difference between the two cohorts $(n=46$ each) is significant we performed a competing risk analysis (Figure 4B). This analysis was done as described in Methods including drop-out patients. Taken together, patients with perioperative ischemia showed significantly more distant or diffuse recurrences than patients without ischemia ( $p=0.0007)$. In contrast, patients of the control cohort (no ischemia) exhibited significantly more local recurrences (Figure $4 \mathrm{~B}, p=$ $0.0011)$.

\section{Progression-free survival and overall survival}

Median time to progression was 253 days for patients with perioperative ischemia and 204 days for the control cohort (Figure 5A, $p=0.46$ ). Median OS was 518 days for patients with perioperative ischemia and 544 days for the control cohort (Figure 5B, $p=0.65$ ). 


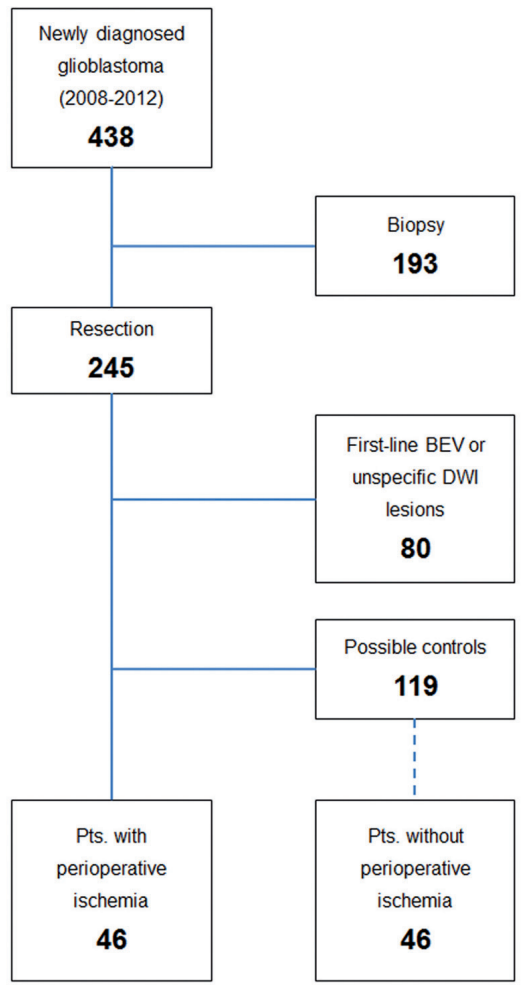

Figure 1: Patient selection. Patient selection of the current study is shown. 193 patients were biopsied and therefore excluded. Another 80 patients were excluded due to small and unspecific lesions of restricted diffusion or BEV first line treatment. In 46 patients we identified unequivocal new perioperative cerebral ischemia on postoperative MRI, whereas 119 did not show lesions of restricted diffusion. These patients served as selection cohort for the 1:1 matched pairs analysis as described in methods.

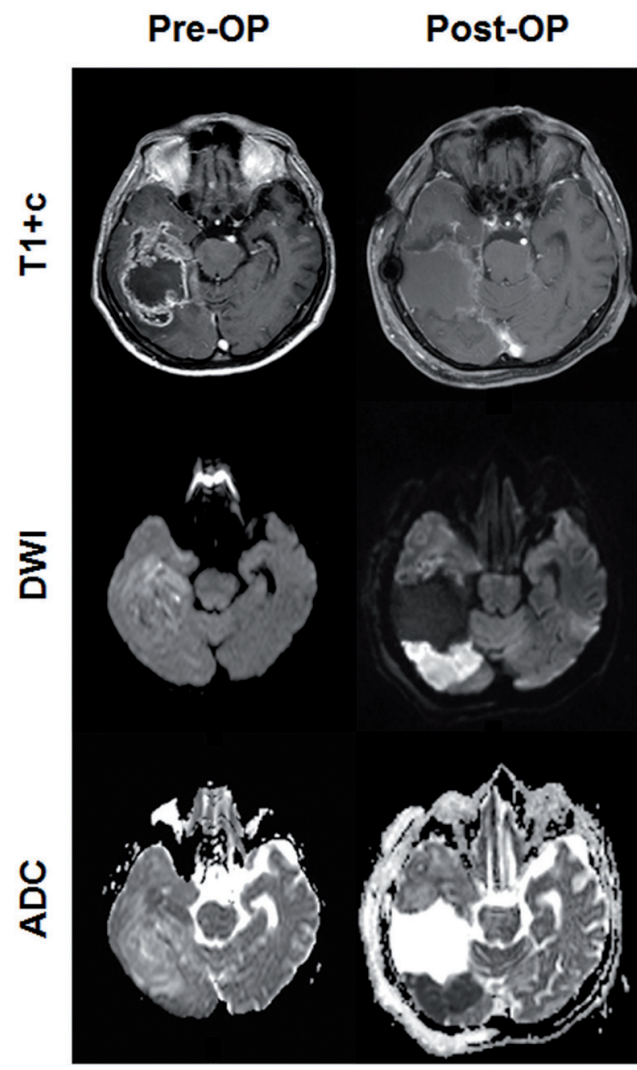

Figure 2: Perioperative ischemia. Representative pre- and postoperative ( $<72 \mathrm{~h}$ after surgery) MRI scans from a patient with perioperative ischemia ARE shown. 

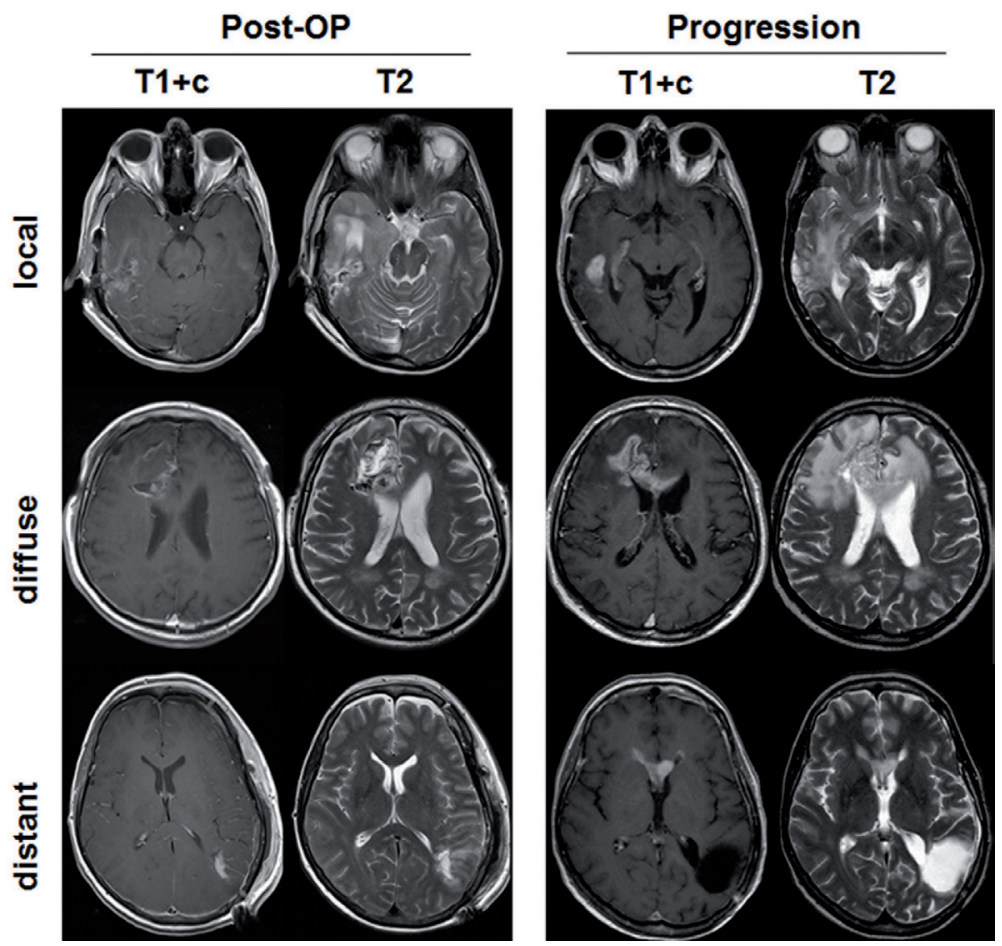

Figure 3: Represantative images for patterns of progression. MRI scans for the three types of progression (local, diffuse, distant), as predefined in Methods, are shown. The first two columns show the postoperative situation on contrast enhanced T1 sequences (T1+c) and T2 weighted sequences. The last two columns show the corresponding scans at the time of first progression.

A

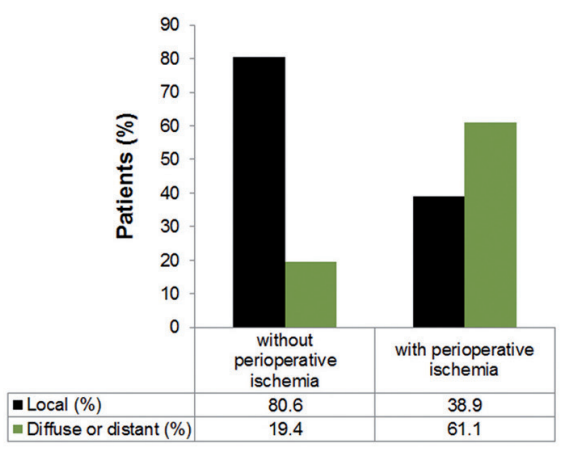

B

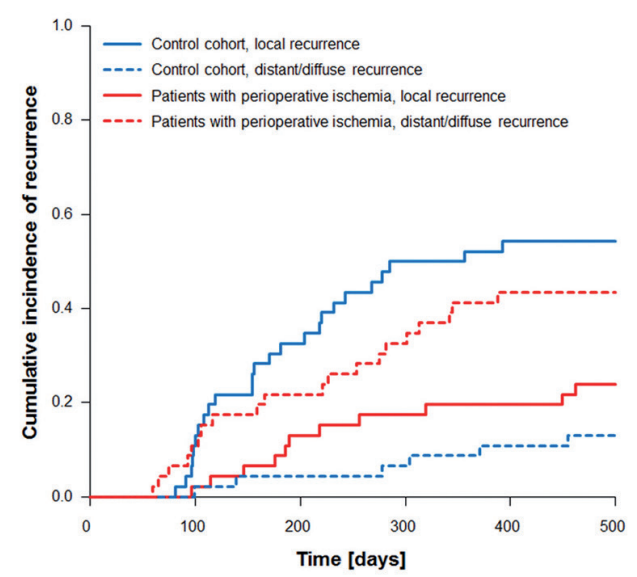

Figure 4: Frequency of patterns of progression. A. shows the distribution of local and infiltrative recurrences (diffuse or distant) for patients with and without perioperative ischemia. B. shows the cumulative incidence of recurrences over time (competing risk analysis). 


\section{DISCUSSION}

This study reveals a previously unrecognized association of perioperative cerebral ischemia with distant or diffuse patterns of progression in patients with newly diagnosed glioblastoma.

The shift towards infiltrative patterns of progression in glioblastoma patients with relevant perioperative ischemia was substantial compared to the matched pair patient cohort and historical data.[1]

The percentage of diffuse or distant recurrences at first relapse was $19.4 \%$ in our control cohort and $61.1 \%$ in the cohort of patients with perioperative ischemia.

A

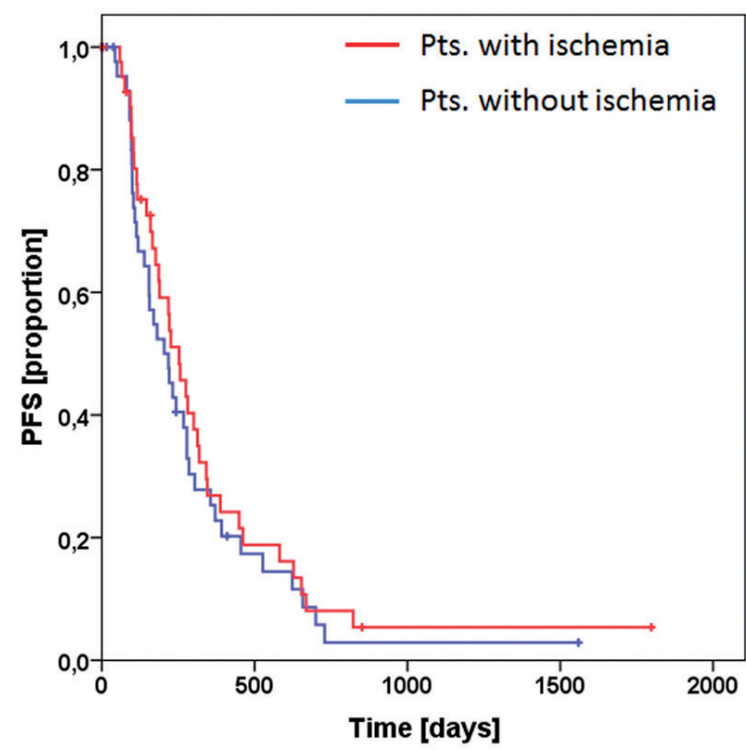

B

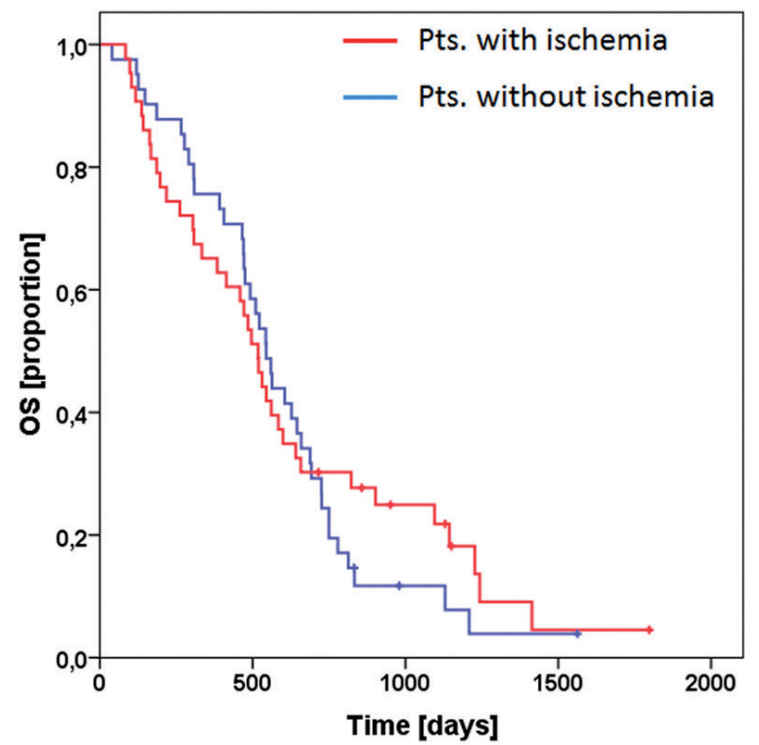

Figure 5: Survival analysis. PFS for patients with perioperative ischemia (red line) and matched controls (blue line) are shown in A. OS for both groups is shown in B. Tick marks indicate censored patients.
Accordingly, the percentage of local recurrences dropped from $80.6 \%$ in the control cohort to $38.9 \%$ in the stroke cohort (Figure 4A). The distribution between local $(80.6 \%)$ and infiltrative (19.4\%) recurrences in our control cohort nicely matches with previous data. Chamberlain reported on $80 \%$ local relapses (64 of 80 patients) at first recurrence in glioblastoma patients.[1]

Interestingly, the increase in infiltrative growth in the ischemia group did not translate into worse progression free or overall survival (Figure 5).

Limitations of this study are (i) the small number of patients and (ii) the retrospective character. Nevertheless, the extent of the increase in infiltrative patterns of progression compared to our matched pair cohort and to historical data is impressive. The exact underlying mechanism for this increase cannot be clarified by this study. However, stroke is the best understood paradigm for cerebral ischemia. It appears likely that acute sublethal hypoxia during perioperative cerebral ischemia alters the tumor biology of affected residual glioma cells. Alternatively, cytokines released from ischemic tissue or systemic factors induced during cerebral ischemia may be involved. Hepatocyte growth factor (HGF) and Tissue Factor (TF) are two examples of a number of cytokines that show increased serum levels after cerebral ischemia and are known to induce glioma cell migration. [6, 20-25]

Our results match with findings in our previous study on BEV induced stroke-like lesions.[26] Patients that developed these lesions with restricted diffusion and $\mathrm{T} 1$ hyperintensities showed an increase of distant recurrences $(41 \%$ vs. $18 \%$ in the cohort without strokelike lesions). The histological evaluation showed extensive calcified necrosis in 4 out of 4 patients.[26] Furthermore, we found an increased HIF-1-alpha expression in an index patient with a lesion of restricted diffusion.[13, 14] We therefore hypothesized a true antiangiogenic effect with severe hypoxia leading to these stroke-like lesions and subsequent increase in distant relapses. Supporting this, preclinical and clinical studies have shown a BEV-induced increase in the expression of hypoxia inducible factor- $1 \alpha$ (HIF-1 $\alpha$ ) or its target genes.[9-14]

In contrast, the majority of studies analyzing patterns of recurrence in glioblastoma patients receiving BEV have not found an increase in distant recurrences. However, these studies are based on low numbers of patients or have not discriminated responders from nonresponders.[1, 18, 19]

There is no doubt that hypoxia is a key driver for infiltrative tumor growth in experimental glioma models. However, evidence that this is true in glioma patients is missing. In the current study we took advantage of the fact that some glioma patients suffer a cerebral ischemia during resection and thereby presumably exposing residual tumor cells to hypoxia. The magnitude of the increase of infiltrative tumor growth from approx. $20 \%$ to $60 \%$ is impressive. Beyond being the first clinical study 
Table 1: Patient characteristics.

\begin{tabular}{|l|l|l|}
\hline Characteristics & $\begin{array}{l}\text { Patients with } \\
\text { perioperative ischemia } \\
(\mathrm{n}=46)\end{array}$ & $\begin{array}{l}\text { Matched pairs without } \\
\text { perioperative ischemia } \\
(\mathrm{n}=46)\end{array}$ \\
\hline General & & \\
\hline Age, median (range) & $62(25-79)$ & $63(36-79)$ \\
\hline Female, $(\mathrm{n})$ & $39.1 \%(18)$ & $39.1 \%(18)$ \\
\hline KPS, median (range) & $80 \%(20-100)$ & $90 \%(40-100)$ \\
\hline Surgery & & \\
\hline GTR or STR, (n) & $84.8 \%(39)$ & $82.6 \%(38)$ \\
\hline Partial resection, (n) & $15.2 \%(7)$ & $15.2 \%(7)$ \\
\hline Unknown & $0 \%(0)$ & $2.2 \%(1)$ \\
\hline Histology & & \\
\hline Glioblastoma, $(\mathrm{n})$ & $100 \%(46)$ & $100 \%(46)$ \\
\hline MGMT-Promoter & & \\
\hline Methylated & $45.7 \%(21)$ & $41.3 \%(19)$ \\
\hline Not methylated & $45.7 \%(21)$ & $47.8 \%(22)$ \\
\hline Unknown & $8.7 \%(4)$ & $10.9 \%(5)$ \\
\hline Days to treatment, median (range) & $28(12-66)$ & $30(13-345)$ \\
\hline Adjuvant treatment & & \\
\hline Radiochemotherapy, (n) & $71.7 \%(33)$ & $71.7 \%(33)$ \\
\hline Radiotherapy, $(\mathrm{n})$ & $10.9 \%(5)$ & $10.9 \%(5)$ \\
\hline Temozolomide, $(\mathrm{n})$ & $4.3 \%(2)$ & $4.3 \%(2)$ \\
\hline Unknown, $(\mathrm{n})$ & $10.9 \%(5)$ & $10.9 \%(5)$ \\
\hline No therapy, (n) & $2.2 \%(1)$ & $2.2 \%(1)$ \\
\hline
\end{tabular}

demonstrating an effect of cerebral ischemia on tumor growth patterns our results are clinically relevant. Diffuse and distant relapses limit therapeutic options at recurrence and impair patients' performance status. Therefore, the cautiousness during resections is indispensable to avoid the adverse effect of perioperative ischemia. Further, this points out that hypoxia itself or the avoidance of hypoxia could serve as a reasonable therapeutic target to prevent the consequences of hypoxia on tumor biology. Two promising examples are hypoxia-activated drugs and oxygen delivery agents.

In summary, to the best of our knowledge, this is the first study on the influence of perioperative cerebral ischemia on patterns of progression in patients with newly diagnosed glioblastoma. Regarding the impact of hypoxia on tumor biology, this study supports the hypothesis that hypoxia induces infiltrative tumor growth in glioblastoma patients.

\section{MATERIALS AND METHODS}

\section{Study population}

We retrospectively screened postoperative MRI scans for perioperative ischemia in patients with newly diagnosed glioblastoma undergoing tumor resection between January 2008 and December 2012 (Figure 1).
During that period 438 patients had first diagnosis of primary glioblastoma at our center. Out of this cohort 193 patients were biopsied and 245 patients underwent resection. Patients receiving bevacizumab (BEV) as part of their first line treatment and patients with small cerebral ischemia or cerebral ischemia distant from resection cavity were excluded ( 80 patients). Of the remaining 165 patients 46 showed new and relevant ischemia nearby the resection cavity on postoperative MRI (see below). Selection of control patients out of the remaining cohort of 119 patients without perioperative ischemia was done automatically by a software algorithm. The control cohort was generated by a 1:1 matched pairs design based on gender, age and adjuvant treatment. Both cohorts were analyzed for patterns of progression (local, diffuse or distant) by a blinded neuroradiologist. Further, we compared the results with historical data and estimated progression free and overall survival for both groups.

Our institutional review board approved this retrospective study and patients gave their consent for scientific work with clinical data including MRI scans (ethics committee at the University Hospital Frankfurt; reference number 4/09-SNO 01/08).

\section{Magnetic resonance imaging}

Postoperative MRI was performed within $72 \mathrm{~h}$ following tumor resection on a 3 Tesla scanner (Siemens 
Medical AG). The protocol included T1-weighted (T1w) sequences before and after intravenous administration of Gadolinium-containing contrast agent, T2-weighted sequences and DWI-sequences with calculated ADCmaps. To exclude postoperative hemorrhage as a cause of restricted diffusion $\mathrm{T} 2 *$-weighted sequences were applied. DWI-Volume of each slice was approximated by multiplying the area of the ischemic lesions with the slice thickness including the gap between the slices. The volume of the whole ischemic lesion was calculated by adding the volumes of each slice. Only patients showing larger areas of relevant ischemia with high DWI-signal and corresponding low ADC-values nearby the resection cavity were enrolled (DWI-Volume $>3 \mathrm{~cm}^{3}$ ). MRI of a representative patient is shown in Figure 2. A small rim of diffusion restriction around the surgical cavity as often seen on postoperative MRI was not regarded as relevant ischemia. During follow-up the areas of restricted diffusion were assessed with particular attention. As expected, the course of these areas was typical for cerebral ischemia with temporary contrast enhancement and finally ischemic gliosis.

For follow-up all patients had T1-weighted (T1-w) sequences before and after intravenous administration of Gadolinium-containing contrast agent and T2-weighted sequences on a 1.5 Tesla scanner (Philips Medical Systems). Response was determined according to the updated response assessment criteria for high-grade gliomas (RANO).[27]

Patterns of progression were analyzed at first progression by one experienced neuroradiologist (M.W.) who was blinded for the scientific rationale of the analysis. We predefined modified categories as reported by Pope and colleagues and described previously.[18, 26]

Local: Enhancing or non-enhancing tumor at or within $3 \mathrm{~cm}$ of the primary site.

Diffuse: Recurrent tumor extending more than $3 \mathrm{~cm}$ from the primary site with at least $50 \%$ of the margin of the recurrent tumor poorly defined.

Distant: One or more new non-contiguous lesion (enhancing or not) with at least $1 \mathrm{~cm}$ distance to primary site.

Representative images for these three categories are shown in Figure 3. Diffuse and Distant recurrences were regarded as infiltrative patterns of progression and were combined for further analysis.

\section{Statistics}

The generation of the matched pairs design was done with R Version 2.14 (R Foundation for Statistical Computing, Vienna, Austria) using the package Matching (generation of the matched pairs design).

Based on PFS the occurrence of the different types of recurrence (local and distant/diffuse) was analyzed with a competing risk analysis additionally accounting for the competing risks of death before progression, unknown type of progression (insufficient radiology) and censored patient that have not progressed yet. Competing risk analysis was done with R Version 2.14 (R Foundation for Statistical Computing, Vienna, Austria) using the package cmprsk (competing risk analysis).

Progression free survival (PFS) and overall survival (OS) from date of resection were estimated using the Kaplan-Meier method and analyzed via the Log-Rank-test (IBM SPSS Statistics Version 20.0). All tests were twosided and p-values below 5\% were considered significant.

\section{ACKNOWLEDGMENTS}

The Dr. Senckenberg Institute of Neurooncology is supported by the Dr. Senckenberg Foundation and the Hertie Foundation. J.P.S. is "Hertie Professor of Neurooncology".

\section{FUNDING}

There was no funding for the present study.

\section{COMPETING INTERESTS STATEMENT}

None of the authors has to report a conflict of interest.

\section{Editorial note}

This paper has been accepted based in part on peerreview conducted by another journal and the authors' response and revisions as well as expedited peer-review in Oncotarget.

\section{REFERENCES}

1. Chamberlain MC. Radiographic patterns of relapse in glioblastoma. J Neurooncol. 2011; 101:319-323.

2. Jensen RL. Brain tumor hypoxia: tumorigenesis, angiogenesis, imaging, pseudoprogression, and as a therapeutic target. J Neurooncol. 2009; 92:317-335.

3. Semenza GL. Hypoxia-inducible factors: mediators of cancer progression and targets for cancer therapy. Trends Pharmacol Sci. 2012; 33:207-214.

4. Steinbach JP, Wolburg H, Klumpp A, Probst H and Weller M. Hypoxia-induced cell death in human malignant glioma cells: energy deprivation promotes decoupling of mitochondrial cytochrome c release from caspase processing and necrotic cell death. Cell Death Differ. 2003; 10:823-832.

5. Vaupel P. Hypoxia and aggressive tumor phenotype: implications for therapy and prognosis. Oncologist. 2008; 13 Suppl 3:21-26. 
6. Eckerich C, Zapf S, Fillbrandt R, Loges S, Westphal M and Lamszus K. Hypoxia can induce c-Met expression in glioma cells and enhance SF/HGF-induced cell migration. Int J Cancer. 2007; 121(:276-283.

7. Jahangiri A, De Lay M, Miller LM, Carbonell WS, Hu YL, Lu K, Tom MW, Paquette J, Tokuyasu TA, Tsao S, Marshall R, Perry A, Bjorgan KM, Chaumeil MM, Ronen SM, Bergers G, et al. Gene expression profile identifies tyrosine kinase c-Met as a targetable mediator of antiangiogenic therapy resistance. Clin Cancer Res. 2013; 19:1773-1783.

8. Lu KV, Chang JP, Parachoniak CA, Pandika MM, Aghi MK, Meyronet D, Isachenko N, Fouse SD, Phillips JJ, Cheresh DA, Park M and Bergers G. VEGF inhibits tumor cell invasion and mesenchymal transition through a MET/ VEGFR2 complex. Cancer Cell. 2012; 22:21-35.

9. de Groot JF, Fuller G, Kumar AJ, Piao Y, Eterovic K, Ji Y and Conrad CA. Tumor invasion after treatment of glioblastoma with bevacizumab: radiographic and pathologic correlation in humans and mice. Neuro Oncol. 2010; 12:233-242.

10. DeLay M, Jahangiri A, Carbonell WS, Hu YL, Tsao S, Tom MW, Paquette J, Tokuyasu TA and Aghi MK. Microarray analysis verifies two distinct phenotypes of glioblastomas resistant to antiangiogenic therapy. Clin Cancer Res. 2012; 18:2930-2942.

11. Hu YL, DeLay M, Jahangiri A, Molinaro AM, Rose SD, Carbonell WS and Aghi MK. Hypoxia-induced autophagy promotes tumor cell survival and adaptation to antiangiogenic treatment in glioblastoma. Cancer Res. 2012; 72:1773-1783.

12. Keunen O, Johansson M, Oudin A, Sanzey M, Rahim SA, Fack F, Thorsen F, Taxt T, Bartos M, Jirik R, Miletic H, Wang J, Stieber D, Stuhr L, Moen I, Rygh CB, et al. AntiVEGF treatment reduces blood supply and increases tumor cell invasion in glioblastoma. Proc Natl Acad Sci U S A. 2011; 108:3749-3754.

13. Rieger J, Bähr O, Muller K, Franz K, Steinbach J and Hattingen E. Bevacizumab-induced diffusion-restricted lesions in malignant glioma patients. J Neurooncol. 2010; 99:49-56.

14. Rieger J, Bähr O, Ronellenfitsch MW, Steinbach J and Hattingen E. Bevacizumab-induced diffusion restriction in patients with glioma: tumor progression or surrogate marker of hypoxia? J Clin Oncol. 2010; 28:e477; author reply e478.

15. Dutzmann S, Gessler F, Bink A, Quick J, Franz K, Seifert $\mathrm{V}$ and Senft C. Risk of ischemia in glioma surgery: comparison of first and repeat procedures. J Neurooncol. 2012; 107:599-607.

16. Stupp R, Mason WP, van den Bent MJ, Weller M, Fisher B, Taphoorn MJ, Belanger K, Brandes AA, Marosi C, Bogdahn U, Curschmann J, Janzer RC, Ludwin SK, Gorlia T, Allgeier A, Lacombe D, et al. Radiotherapy plus concomitant and adjuvant temozolomide for glioblastoma. N Engl J Med. 2005; 352:987-996.
17. Wick W, Platten M, Meisner C, Felsberg J, Tabatabai G, Simon M, Nikkhah G, Papsdorf K, Steinbach JP, Sabel M, Combs SE, Vesper J, Braun C, Meixensberger J, Ketter R, Mayer-Steinacker R, et al. Temozolomide chemotherapy alone versus radiotherapy alone for malignant astrocytoma in the elderly: the NOA-08 randomised, phase 3 trial. Lancet Oncol. 2012; 13:707-715.

18. Pope WB, Xia Q, Paton VE, Das A, Hambleton J, Kim HJ, Huo J, Brown MS, Goldin J and Cloughesy T. Patterns of progression in patients with recurrent glioblastoma treated with bevacizumab. Neurology. 2011; 76:432-437.

19. Wick A, Dorner N, Schafer N, Hofer S, Heiland S, Schemmer D, Platten M, Weller M, Bendszus M and Wick W. Bevacizumab does not increase the risk of remote relapse in malignant glioma. Ann Neurol. 2011; 69:586592.

20. Brockmann MA, Papadimitriou A, Brandt M, Fillbrandt $\mathrm{R}$, Westphal M and Lamszus K. Inhibition of intracerebral glioblastoma growth by local treatment with the scatter factor/hepatocyte growth factor-antagonist NK4. Clin Cancer Res. 2003; 9:4578-4585.

21. Dutzmann S, Gessler F, Harter PN, Gerlach R, Mittelbronn $\mathrm{M}$, Seifert $\mathrm{V}$ and Kogel $\mathrm{D}$. The pro-migratory and proinvasive role of the procoagulant tissue factor in malignant gliomas. Cell Adh Migr. 2010; 4:515-522.

22. Harter PN, Dutzmann S, Drott U, Zachskorn C, Hattingen E, Capper D, Gessler F, Senft C, Seifert V, Plate KH, Kogel D and Mittelbronn M. Anti-tissue factor (TF9-10H10) treatment reduces tumor cell invasiveness in a novel migratory glioma model. Neuropathology. 2013; 33:515525 .

23. Okazaki H, Beppu H, Mizutani K, Okamoto $\mathrm{S}$ and Sonoda S. Changes in Serum Growth Factors in Stroke Rehabilitation Patients and Their Relation to Hemiparesis Improvement. J Stroke Cerebrovasc Dis. 2014.

24. Sobol AB, Galar M, Mochecka A, Stanczyk L and Kloczko J. [Tissue factor and it's inhibitor in patients up to 50 years of age with ischemic stroke]. Pol Merkur Lekarski. 2001; 10:92-95.

25. Undas A, Slowik A, Gissel M, Mann KG and Butenas S. Active tissue factor and activated factor XI in patients with acute ischemic cerebrovascular events. Eur J Clin Invest. 2012; 42:123-129.

26. Bähr O, Harter PN, Weise LM, You SJ, Mittelbronn M, Ronellenfitsch MW, Rieger J, Steinbach JP and Hattingen E. Sustained focal antitumor activity of bevacizumab in recurrent glioblastoma. Neurology. 2014.

27. Wen PY, Macdonald DR, Reardon DA, Cloughesy TF, Sorensen AG, Galanis E, Degroot J, Wick W, Gilbert MR, Lassman AB, Tsien C, Mikkelsen T, Wong ET, Chamberlain MC, Stupp R, Lamborn KR, et al. Updated response assessment criteria for high-grade gliomas: response assessment in neuro-oncology working group. J Clin Oncol. 2010; 28:1963-1972. 